\title{
Fast System Calibration With Coded Calibration Scenes for Magnetic Particle Imaging
}

\author{
Serhat llbey ${ }^{(0)}$, Can Barış Top ${ }^{(1)}$, Alper Güngör $\left.{ }^{(}\right)$, Tolga Çukur ${ }^{(}$, \\ Emine Ulku Saritas ${ }^{(0}$, and H. Emre Güven ${ }^{(\mathbb{}}$
}

\begin{abstract}
Magnetic particle imaging (MPI) is a relatively new medical imaging modality, which detects the nonlinear response of magnetic nanoparticles (MNPs) that are exposed to external magnetic fields. The system matrix (SM) method for MPI image reconstruction requires a time consuming system calibration scan prior to image acquisition, where a single MNP sample is measured at each voxel position in the field-of-view (FOV). The scanned sample has the maximum size of a voxel so that the calibration measurements have relatively poor signal-to-noise ratio (SNR). In this paper, we present the coded calibration scene (CCS) framework, where we place multiple MNP samples inside the FOV in a random or pseudo-random fashion. Taking advantage of the sparsity of the SM, we reconstruct the SM by solving a convex optimization problem with alternating direction method of multipliers using CCS measurements. We analyze the effects of filling rate, number of measurements, and SNR on the SM reconstruction using simulations and demonstrate different implementations of CCS for practical realization. We also compare the imaging performance of the proposed framework with that of a standard compressed sensing SM reconstruction that utilizes a subset of calibration measurements from a single MNP sample. The results show that CCS significantly reduces calibration time while increasing both the SM reconstruction and image reconstruction performances.
\end{abstract}

Manuscript received December 3, 2018; revised January 25, 2019; accepted January 26, 2019. Date of publication January 31, 2019; date of current version August 30, 2019. This work was supported by the Scientific and Technological Research Council of Turkey (TUBITAK) under Grant 9050103 and Grant 115E677. The work of E. U. Saritas and T. Çukur was supported in part by the Turkish Academy of Sciences through the TUBA-GEBIP 2015 Program, in part by the BAGEP Award of the Science Academy, and in part by the European Molecular Biology Organization through an Installation Grant 3028. (Corresponding author: Serhat Ilbey.)

S. Ilbey and A. Güngör are with the ASELSAN Research Center, 06370 Ankara, Turkey, also with the National Magnetic Resonance Research Center, Bilkent University, 06800 Ankara, Turkey, and also with the Department of Electrical and Electronics Engineering, Bilkent University, 06800 Ankara, Turkey (e-mail: serhatilbey@gmail.com).

C. B. Top and H. E. Güven are with the ASELSAN Research Center, 06370 Ankara, Turkey.

T. Çukur and E. U. Saritas are with the Department of Electrical and Electronics Engineering, Bilkent University, 06800 Ankara, Turkey, also with the National Magnetic Resonance Research Center, Bilkent University, 06800 Ankara, Turkey, and also with the Neuroscience Program, Sabuncu Brain Research Center, Bilkent University, 06800 Ankara, Turkey.

Color versions of one or more of the figures in this article are available online at http://ieeexplore.ieee.org.

Digital Object Identifier 10.1109/TMI.2019.2896289
Index Terms-Magnetic particle imaging, system matrix, compressed sensing, alternating direction method of multipliers, coded calibration scenes, calibration.

\section{INTRODUCTION}

$\mathbf{M}$ AGNETIC particle imaging (MPI) is a medical imaging modality, which uses the nonlinear response of magnetic nanoparticles (MNPs) to image their distribution [1]. Angiography, stem cell tracking, and cancer imaging are some of the potential clinical applications of MPI [2]-[5]. In MPI, a static magnetic field called the selection field (SF) generates a field free region (FFR) in the field-of-view (FOV). A timevarying magnetic field called the drive field (DF) generates a time-varying magnetization of the MNPs inside the FFR. A signal is induced in the receive coils by this magnetization response. MNP response quickly decreases with increasing distance from the FFR, reaching saturation.

There are two main approaches for image reconstruction in MPI [6]: model-based and measurement-based methods. In the model-based methods, the system matrix (SM) or the point spread function (PSF) is estimated analytically based on the physical model of the MNPs and the hardware of the system [7], [8]. On the other hand, the measurement-based methods require imaging an MNP sample with known concentration and shape to obtain the SM or PSF [1], [9]. For instance, to form the SM, an MNP sample is scanned at each voxel position in the FOV, and the Fourier transform of the received signal is recorded at each position. After this system calibration procedure, one can solve a linear system of equations for image reconstruction using regularization or optimization algorithms such as the algebraic reconstruction technique [10], nonnegative fused LASSO [11], and Alternating Direction Method of Multipliers (ADMM) [12]. The explicit calibration procedure in the measurement-based methods increases reliability against system imperfections compared to the model-based methods [12]. This is especially important for systems using complex trajectories (e.g., Lissajous trajectory) that involve fast rotation of the magnetization vector [13]. However, the additional measurements introduce a scan time cost, e.g., even for a small FOV with $20 \times 20 \times 20$ voxels, the calibration would take nearly 3 hours with each position captured in 1.3 seconds [14]. As this work focuses on improving the measurement-based methods, SM will refer to a system matrix formed after a calibration process. 
In the literature, there are Compressed Sensing (CS) methods to mitigate the cost of long calibration scans [15]-[18]. The motivation for CS is that the SM has highly sparse or compressible representations in several known transform domains [15], [16]. As such, a previously proposed CS method (hereinafter, referred to as the "standard CS method") was shown to successfully recover the SM using as low as $10-20 \%$ of the full set of calibration measurements, using a $5.5 \mathrm{~T} / \mathrm{m} \mathrm{SF}$ gradient and a two-dimensional (2D) Lissajous trajectory with $18 \mathrm{mT}$ DF strength [17]. The quality of the SM reconstruction inevitably degrades with measurement sets reduced to fit within clinically acceptable calibration times. Besides that, a recently proposed hybrid approach shows that performing calibration in a separate device emulating the MPI system enhances the SM reconstruction quality [14]. While promising, this approach has challenges in practice, since it requires explicit knowledge of the magnetic field distribution across time and space. A recently proposed approach enhances the signal-to-noise ratio (SNR) of SM measurements by decreasing the SF gradient and increasing the grid size [19]. However, a large calibration time is still required as this method does not involve an undersampling of SM.

In this work, we introduce a Coded Calibration Scene (CCS) framework for fast system calibration. We implement CCS as a calibration phantom that includes multiple MNP samples placed at random positions, with a size larger than or equal to the imaging FOV. One of the main advantages of CCS is that it yields a significantly higher SNR than the standard calibration measurement performed in a single location. Moreover, the received signal induced from the MNPs at multiple positions increases the information content of each calibration measurement [20].

Using simulations, we thoroughly analyze the performance of the CCS framework based on several practical implementations relevant to realistic imaging scenarios. We use ADMM to rapidly reconstruct the SM using CCS measurements [21]. We show that CCS significantly improves image quality relative to the standard CS method [17], even with highly accelerated calibrations. We also investigate the effects of CCS filling rate, number of measurements, SNR, and positioning error on the accuracy of the SM reconstruction via extensive simulations.

\section{Methods}

Here, we define $N_{y}$ and $N_{z}$ as the number of pixels along the $y$ - and z-directions, respectively, "measurement rate" $(\delta)$ for the calibration procedure as the ratio of the number of measurements to the number of pixels in the FOV $\left(N=N_{y} \times N_{z}\right)$, "filling rate" $(v)$ of a CCS as the number of pixels filled with MNP samples divided by $N$, and $M$ as twice the number of used frequency components of the signal spectrum (due to separating and appending real and imaginary parts).

\section{A. MPI System Calibration With Compressed Sensing}

A linear set of equations represents the imaging problem in MPI [1]:

$$
b=A x+n,
$$

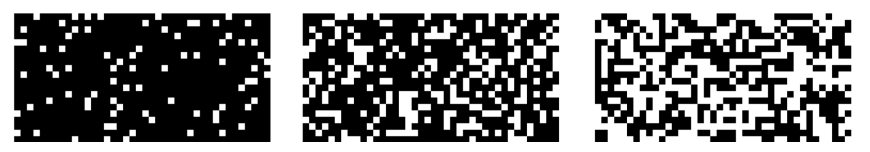

Fig. 1. Examples of the Multi-CCS (M-CCS) implementation that are randomly created with $0.1,0.3$, and 0.5 filling rates $(\nu)$, from left to right. These CCSs have dimensions $20 \mathrm{~mm} \times 10 \mathrm{~mm}$ with $40 \times 20$ pixels.

where $A \in \mathbb{R}^{M \times N}$ is the full SM, $x \in \mathbb{R}^{N \times 1}$ is the vectorized representation of the MPI image to be reconstructed, $b \in \mathbb{R}^{M \times 1}$ is the measurement vector including additive noise $n \in \mathbb{R}^{M \times 1}$. Conventionally, one obtains the SM by measuring a single MNP sample at each voxel position in the FOV. The SM is sparse in discrete Chebyshev transform, discrete cosine transform (DCT), and discrete Fourier transform domains, and the CS methods can be used to decrease the number of calibration measurements. The standard CS method suggests randomly selecting voxel positions in the FOV grid and moving an MNP sample to those positions to take calibration data. As such, one reconstructs the SM using convex optimization algorithms utilizing the sparsity property of the SM [17]. Accordingly, the following optimization problem is solved:

$$
\begin{gathered}
\underset{A}{\operatorname{argmin}}\left\|D A^{T}\right\|_{1} \\
\text { subject to }\left\|A C-A_{c}\right\|_{F}<\epsilon_{c},
\end{gathered}
$$

where $D \in \mathbb{R}^{N \times N}$ is the matrix representation of the DCT and $A_{c} \in \mathbb{R}^{M \times \delta N}$ is the matrix of undersampled calibration measurements. $C \in \mathbb{R}^{N \times \delta N}$ is the masking matrix that chooses the columns of $A$ corresponding to the positions for which there are calibration measurements. $\|\cdot\|_{F}$ is the Frobenius norm of a matrix and $\|\cdot\|_{1}$ denotes the sum of absolute values of all elements of a matrix in this paper.

\section{B. MPI System Calibration With Coded Calibration Scenes}

In this work, we propose the use of CCSs for SM calibration. In the standard CS method, each column of the $C$ matrix in Eqn. (2) has only one non-zero entry at the position of the MNP sample for the corresponding calibration measurement. Note that an identity $C$ matrix corresponds to measuring the response of the MNP sample at each voxel position, which is the conventional calibration procedure without CS or undersampling. For that case, there is no need to solve the optimization problem in Eqn. (2). In contrast, multiple MNP samples are present in each CCS. Therefore, each column of the $C$ matrix corresponds to the nanoparticle distribution of a single CCS.

This work proposes five different approaches for CCS-based calibration, as described below:

1) Multi-CCS (M-CCS): This is the basic implementation of CCS. Multiple CCSs are used for calibration. Each CCS has the size of the FOV. For each CCS, we randomly place voxelsized MNP samples on the FOV grid with a certain filling rate, $v$. For a given $\delta$ and $\nu$, we use the $C$ matrix with the smallest mutual coherence among all possible set of matrices to decrease the coherency between the CCSs used in the calibration [22]. We show examples of M-CCS with different $v$ values in Fig. 1. For calibration measurements, we place a CCS in the FOV, measure, and then replace by another CCS. 


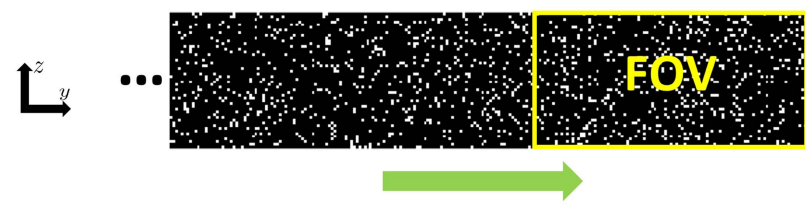

(a)

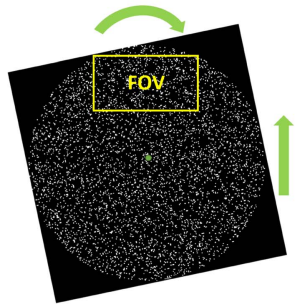

(b)

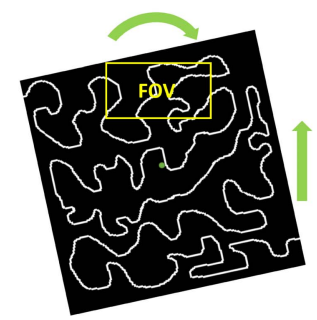

(c)
Fig. 2. Practical implementations of CCSs. a) Sliding CCS (S-CCS) filled randomly with MNPs and slided through the FOV. Number of measurements and the amount of shift between consecutive measurements determine the length of the CCS. b) Rotating CCS (R-CCS) filled randomly with MNPs and rotated about its center. R-CCS can be slided after a $360^{\circ}$ turn, and rotated about a different axis, which is named as a Rotating and Sliding CCS (RS-CCS). c) Rotating and Sliding Contiguous CCS (RSC-CCS), where the MNPs are contiguously distributed for a more practical implementation. $\nu$ of these examples is 0.1 .

2) Sliding CCS (S-CCS): This is an extended size CCS, which can be slided through the FOV. This way, the need for multiple CCS preparations and replacements is eliminated. We show an S-CCS in Fig. 2a. We slide the S-CCS along the $\mathrm{y}$-direction by a predetermined number of grids before each calibration measurement.

Let $C_{S} \in \mathbb{R}^{N_{z} \times L}$ be the mathematical representation of S-CCS where $L$ is number of the grids in the CCS along the y-direction, $C_{i} \in \mathbb{R}^{N_{z} \times N_{y}}$ be the $2 \mathrm{D}$ representation of the $i^{\text {th }}$ column of $C$, and $S_{\Delta_{y}} \in \mathbb{R}^{L \times N_{y}}$ be the shift matrix that shifts $C_{S}$ in the y-direction by $\Delta_{y}$ grids. Then, one can show that

$$
C_{i}=C_{S} S_{\Delta_{y}},
$$

where

$$
S_{\Delta_{y}}=\left[\begin{array}{c}
0_{l \times N_{y}} \\
S_{T_{\left(N_{y}+1\right) \times N_{y}}} \\
0_{k \times N_{y}}
\end{array}\right] .
$$

Here, $0_{i \times j}$ is the zero matrix of size $i \times j, l=L-N_{y}-k-1$, $k$ is the maximum non-negative integer satisfying $\Delta_{y}=k+\Delta$, where $\Delta \in(0,1)$ allows off-grid shifts of CCS, and

$$
S_{T}=\left(\begin{array}{ccccccc}
\Delta & 0 & 0 & 0 & 0 & \ldots & 0 \\
1-\Delta & \Delta & 0 & 0 & 0 & \ldots & 0 \\
0 & 1-\Delta & \Delta & 0 & 0 & \cdots & 0 \\
\vdots & \vdots & \vdots & \vdots & \vdots & \ddots & \vdots \\
0 & 0 & 0 & 0 & 0 & 1-\Delta & \Delta \\
0 & 0 & 0 & 0 & 0 & 0 & 1-\Delta
\end{array}\right) .
$$

3) Rotating CCS (R-CCS): In R-CCS, we rotate a single CCS larger than the FOV during calibration measurements. We show an R-CCS example in Fig. 2b, also depicting the size and position of the FOV. We position the FOV as far away from the center of R-CCS as possible, so that rotations cause maximal change in the MNP distribution that fall within the FOV. While the calibration phantom is implemented on a rectangular grid, rotation naturally leads to off-grid MNP distributions. When the phantom is rotated, we analytically calculate the percentage of MNPs that fall on each grid, and generate the $C$ matrix using this information. Note that the elements of $C$ are no longer binary in this case (e.g., the R-CCS in Fig. 2b have non-binary pixel values).

First, we calculate the coordinates of the central positions of the MNP samples in the scene. Let $X_{\theta} \in \mathbb{R}^{N_{R} \times 2}$ be the matrix that holds the $2 \mathrm{D}$ coordinates of the MNPs when the R-CCS is $\theta$ degrees rotated, where $N_{R}$ is number of grids in the CCS. Then one can show that

$$
X_{\theta}=X_{0^{\circ}} R_{\theta},
$$

where

$$
X_{0^{\circ}}=\left[\begin{array}{cc}
x_{1} & y_{1} \\
\vdots & \vdots \\
x_{N_{R}} & y_{N_{R}}
\end{array}\right], \quad \text { and } R_{\theta}=\left[\begin{array}{cc}
\cos (\theta) & -\sin (\theta) \\
\sin (\theta) & \cos (\theta)
\end{array}\right] \text {. }
$$

Then, for a given $\theta$, the amount of MNPs inside each grid is computed based on these coordinates.

4) Rotating and Sliding CCS (RS-CCS): RS-CCS is a variation of R-CCS, where we shift the scene after a full $\left(360^{\circ}\right)$ turn, and we take further measurements around the new rotation axis (Fig. 2b) [21]. The advantage of this implementation is that one can perform the calibration using a smaller sized CCS compared to S-CCS and R-CCS.

5) Rotating and Sliding Contiguous CCS (RSC-CCS): This, in our view, is a more practical implementation of RS-CCS, in which we distribute the MNP samples in a contiguous fashion (Fig. 2c). This implementation allows filling (and emptying) the CCS with nanoparticles easily, e.g., by using a capillary tube filled with MNP solution.

There are a number of design parameters for the CCS implementations such as $v, \delta$, the step size for sliding $\left(\Delta_{y}\right)$, and the angular step size $\left(\Delta_{\theta}\right)$ for R-CCS and its variations. We analyze the effects of these parameters on the SM reconstruction performance via simulations. We also compare the MPI images reconstructed using the SMs obtained by the proposed CCS implementations.

\section{MPI Simulations}

We evaluated the proposed methods using an in-house MPI simulator. We set the magnetic field gradients to $1.25 \mathrm{~T} / \mathrm{m}$ and $2.5 \mathrm{~T} / \mathrm{m}$ in the $\mathrm{y}$ - and z-directions, respectively. We used a Lissajous trajectory with $f_{y}=26.042 \mathrm{kHz}$ and $f_{z}=$ $25.253 \mathrm{kHz}$ frequencies to scan the field free point at the entire FOV [23]. We assumed a mono-disperse MNP model with $25 \mathrm{~nm}$ diameter [24], [25] and $37{ }^{\circ} \mathrm{C}$ temperature. For the imaging analyses, we performed simulations for a FOV of $50 \mathrm{~mm} \times 25 \mathrm{~mm}(100 \times 50$ pixels $)$ with $31.25 \mathrm{mT} \mathrm{DF}$ amplitudes. For the SM reconstruction performance analyses, 


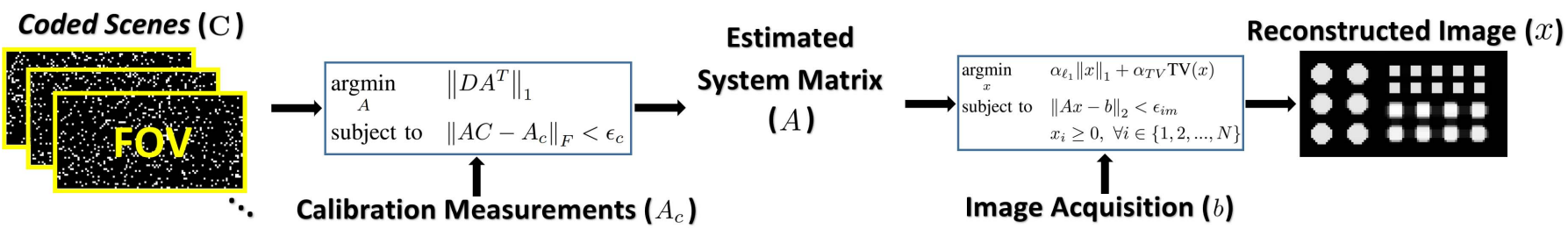

Fig. 3. The flowchart for CCS-based MPI system calibration and image reconstruction. The calibration measurements $\left(A_{C}\right)$ are taken using the CCSs (represented as $C$ in the problem formulations). First, we use ADMM, by minimizing the $\ell_{1}$-norm of the DCT of the SM, subject to the data fidelity constraint, to estimate the full system matrix $(A)$ with these measurements. Next, the image acquisition starts. With the estimated $A$ and imaging measurements $b$, we use ADMM by minimizing the weighted sum of the total variation and the $\ell_{1}$-norm of the reconstructed image with data fidelity and nonnegativity constraints to reconstruct the MPI image.

a smaller FOV with $20 \mathrm{~mm} \times 10 \mathrm{~mm}$ was used to accelerate the extensive analyses targeted in this work. For that case, the FOV was discretized into a $40 \times 20$ pixel grid, and the corresponding DF amplitudes were $12.5 \mathrm{mT}$ for both $\mathrm{y}$ - and z-directions.

We calculated the received signal using the following equation [26]:

$$
u(t)=\int_{F O V}-\mu_{0} p^{R}(r) \cdot \frac{\partial \bar{m}(r, t)}{\partial t} \bar{x}(r) d^{3} r,
$$

where $\bar{x}$ is the three-dimensional (3D) MNP distribution in the FOV,$p^{R}$ is the sensitivity profile of the receive coils, modeled as uniform, $\mu_{0}$ is the free space magnetic permeability, and $\bar{m}$ is the average magnetic moment of the MNPs [26].

The sampling rate of the received signal was $20 \mathrm{MHz}$. We stored and used the $30 \mathrm{kHz}-1 \mathrm{MHz}$ band of the signal. The additive white Gaussian noise was generated with the built-in wgn function of MATLAB. We used the signal power obtained with a single-voxel MNP sample as the reference when calculating the standard deviation of the noise at different SNR levels.

\section{System Matrix Reconstruction}

To solve the problem defined in Eqn. (2), which is a simple $\ell_{1}$-norm based problem that has been discussed thoroughly in the literature [27]-[29], we used ADMM for both the standard CS and the proposed CCS implementations [21]. ADMM is an optimization algorithm that solves a given convex optimization problem by augmenting a Lagrangian term and dividing it into two easier sub-problems. In this study, we use convex functions as objectives and intersection of convex sets as constraints. For these types of convex problems, ADMM is proven to converge [30]. Moreover, ADMM requires the existence of the exact or an approximate solution of the sub-problems [30], and we previously presented details of the exact solutions of each sub-problem in [12]. In Fig. 3, we show the flowchart for the system calibration and SM reconstruction procedure. In standard CS reconstruction, we used a single-voxel MNP sample instead of coded scenes.

We analyzed the effects of CCS design parameters on SM reconstruction performance via the following simulations:

1) First, we analyzed the effect of $v$ on the SM reconstruction for M-CCS at different measurement rates $(\delta=0.5,0.2$, 0.1 , and 0.05$)$ and various SNR levels $(0 \mathrm{~dB}, 10 \mathrm{~dB}, 20 \mathrm{~dB}$, $30 \mathrm{~dB}$, and without added noise). The simulated $v$ values were
$1 / 800,0.1,0.3,0.5,0.7,0.9$, and $799 / 800$. Note that for the $40 \times 20$ pixel FOV used in these simulations, $v=1 / 800$ corresponds to a CCS with a single-voxel MNP sample, which is equivalent to the standard CS method. The dual case is $v=799 / 800$, in which all voxels except for one voxel are filled with MNP samples. We also performed a similar analysis for the full rotation of R-CCS at $\delta=0.45,0.18,0.1$, and 0.05 , corresponding to $\Delta_{\theta}=1^{\circ}, 2.5^{\circ}, 4.5^{\circ}$, and $9^{\circ}$, respectively. The simulated $v$ values were $0.1,0.3,0.5,0.7$, and 0.9 . The size of the R-CCS was $50 \mathrm{~mm} \times 50 \mathrm{~mm}$ (corresponding to $100 \times 100$ voxels), and the distance between the FOV center and the rotation center was $17.5 \mathrm{~mm}$ in these simulations.

2) The frequency components of the SM can be formulated with respect to the mixing factors $m_{y}$ and $m_{z}$ as follows [15], [26]:

$$
k=m_{y}(\kappa+1)+m_{z} \kappa,
$$

where $k$ is the frequency index of the SM, and $\kappa /(\kappa+1)=$ $f_{z} / f_{y}$. We compared the SM reconstruction performances of M-CCS and R-CCS with the standard CS method qualitatively with respect to different mixing orders.

3) For S-CCS, we analyzed the effect of $\Delta_{y}$ on the SM reconstruction for $\Delta_{y}$ values ranging from 1 to 40 grids. Similarly for R-CCS, we analyzed the effect of $\Delta_{\theta}$ with $\Delta_{\theta}$ ranging from $0.5^{\circ}$ to $5^{\circ}$. Both analyses were performed for $\delta=0.2$ at $v=0.1$ and $10 \mathrm{~dB}$ SNR.

4) One may not know the position of S-CCS or R-CCS precisely during a continuous sliding/rotation movement. For S-CCS with $\Delta_{y}=40$ grids (i.e., the equivalent of M-CCS), we analyzed the effect of positioning error on the SM reconstruction for random errors drawn from a uniform distribution with maximum error ranging between $1 \%$ and $20 \%$ of the length of 1 grid. For R-CCS, we analyzed the effect of angular positioning error on the SM reconstruction for errors ranging from $0.01^{\circ}$ to $0.2^{\circ}$. For each measurement, the positioning error was drawn from a discretized uniform distribution with $0.01^{\circ}$ steps to reduce the computational cost of Monte-Carlo simulations.

5) For a limited system calibration duration, an important question is how many different CCSs one should measure for optimal performance for a given measurement SNR and duration. Hence, we analyzed the trade-off between using the time budget to increase the SNR (via averaging) and varying the effective CCS. We used an S-CCS with $\Delta_{y}=40$ grids. We assumed that the time required for sliding between 


\section{亡}

Fig. 4. Numerical phantom used in the simulations, with an overall size of $50 \mathrm{~mm} \times 25 \mathrm{~mm}$. The disks have $5 \mathrm{~mm}$ (left) and $4 \mathrm{~mm}$ (bottom right) diameters. The squares (top right) have $2.5 \mathrm{~mm}$ side lengths.

consecutive measurements is 1 second, the measurement time for a single Lissajous cycle is 1 millisecond, the SNR is $-30 \mathrm{~dB}$, and $v=0.1$. We fixed the total calibration time to $\sim 320$ seconds. We performed a similar analysis for R-CCS, with the assumption that 1 second is required for mechanical rotation between consecutive measurements. $\Delta_{\theta}$ for rotation was set according to the number of calibration measurements to complete 1 full turn, fixing the total calibration time to $\sim 360$ seconds. For example, for 144 measurements, $\Delta_{\theta}$ was $2.5^{\circ}$. Again, we assumed that a single Lissajous cycle is 1 millisecond, the SNR is $-30 \mathrm{~dB}$, and $v=0.1$.

To quantitatively assess the performance of the SM reconstructions, we calculated the estimation errors of the reconstructed SMs using normalized root-mean-squared error (nRMSE), defined as:

$$
\mathrm{nRMSE}=20 \log _{10}\left(\frac{\sqrt{\frac{\sum_{i=1}^{M} \sum_{j=1}^{N}\left(A_{i j}-\tilde{A}_{i j}\right)^{2}}{M N}}}{\sigma_{\tilde{A}}}\right),
$$

where scalars $M$ and $N$ are the number of rows and columns of $A$, respectively. $\tilde{A}$ is the ideal SM obtained with a full calibration measurement without noise and $\sigma_{\tilde{A}}$ is the standard deviation of the elements of $\tilde{A}$.

We ran MC simulations until convergence for each CCS analysis, where we defined convergence as reaching a change in the mean nRMSE level below $-60 \mathrm{~dB}$ between consecutive iterations. $200 \mathrm{MC}$ simulations were sufficient to reach convergence for all analyses.

\section{E. Image Reconstruction}

For the image reconstruction, we solved the following problem with ADMM [12], [31]:

$$
\begin{aligned}
\underset{x}{\operatorname{argmin}} & \alpha_{\ell_{1}}\|x\|_{1}+\alpha_{T V} \operatorname{TV}(x) \\
\text { subject to } & \|A x-b\|_{2}<\epsilon_{i m} \\
& x_{i} \geq 0, \quad \forall i \in\{1,2, \ldots, N\},
\end{aligned}
$$

where $\alpha_{\ell_{1}}$ and $\alpha_{T V}$ are scalar weights of the $\ell_{1}$-norm and total variation (TV) terms, respectively, $\epsilon_{i m}$ is the bound on the data fidelity error, and $x_{i}$ is the voxel value at the $i^{\text {th }}$ position of $x$. We define the problem in a way that a weighted sum of the $\ell_{1}$-norm and TV of the reconstructed images is minimized. Moreover, we also used a nonnegativity constraint, as the MNP density cannot be negative [12], [32]. We previously presented the details of image reconstruction with ADMM algorithm in [12]. Defining the problem with TV and $\ell_{1}$-norm cost functions is highly suitable for the medical imaging applications targeted by MPI, since the images are (a)

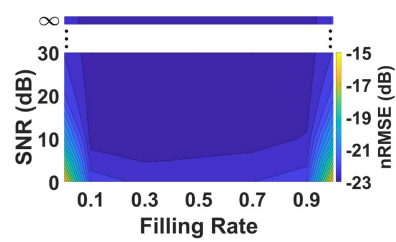

(c)

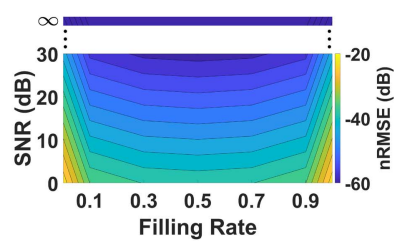

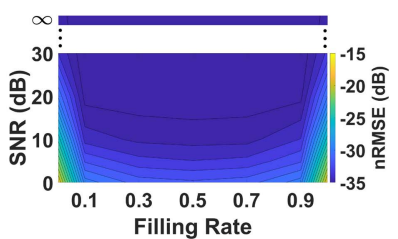

(b)

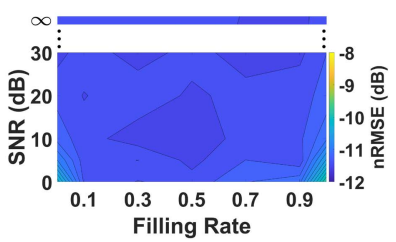

(d)
Fig. 5. Contour plots of the nRMSE of the estimated system matrices for different filling rates $(\nu)$ and $\delta$ values for M-CCS. For $\nu$, we used the following values: $1 / 800,0.1,0.3,0.5,0.7,0.9$, and $799 / 800$. a) $\delta=0.5$. b) $\delta=0.2$. c) $\delta=0.1$. d) $\delta=0.05$.

block-wise contiguous and sparse. For example, the images of blood vessels have these properties naturally.

We present the schematic describing the image reconstruction in Fig. 3 for M-CCS. We show the $100 \times 50$ pixel numerical phantom used in the study in Fig. 4. This phantom has disk objects with $4 \mathrm{~mm}$ and $5 \mathrm{~mm}$ diameters, and squares with side lengths of $2.5 \mathrm{~mm}$.

To analyze the image reconstruction performance, we tested three different scenarios: $\delta=0.2$ and $10 \mathrm{~dB}$ SNR, $\delta=0.2$ and $0 \mathrm{~dB} \mathrm{SNR}$, and $\delta=0.1$ and $0 \mathrm{~dB}$ SNR. We utilized a practical filling rate of $v=0.1$ for all CCSs and for all scenarios. For S-CCS, $\Delta_{y}$ was 5 grids in the y-direction. The sizes of RS-CCS and RSC-CCS were $250 \times 250$ voxels, corresponding to a 12.5 times larger area than the FOV. The distance between the center of the FOV and the rotation center was $45 \mathrm{~mm}$ in the z-direction, and $\Delta_{\theta}$ was $1^{\circ}$. After each full turn, we slided the CCS 30 grids in the z-direction twice for the $\delta=0.2$ case. This corresponds to 1080 measurements $(\delta=0.216)$ in total with three full turns of the CCS. On the other hand, for $\delta=0.1$ case, we used $1.5^{\circ}$ angle-steps and slided the CCS once for 40 grids in the z-direction. This corresponds to 480 measurements $(\delta=0.096)$ in total with two full turns of the CCS.

In addition, for S-CCS, we analyzed the effect of $\Delta_{y}$ on the reconstructed images for the cases of $\Delta_{y}=1,5$, and 10 grids. For this analysis, the other parameters were $\delta=0.2$, $10 \mathrm{~dB}$ SNR, and $v=0.1$.

To compare the quality of the normalized reconstructed images, we calculated the structural similarity index (SSIM) [33] and Peak SNR (PSNR) values using the built-in ssim and psnr functions of MATLAB with the default parameters.

\section{Results}

\section{A. Effects of Filling Rate (v), Measurement Rate $(\delta)$, and SNR}

We analyzed the robustness of the SM reconstruction for M-CCS and R-CCS, with the contour plots of the nRMSE 


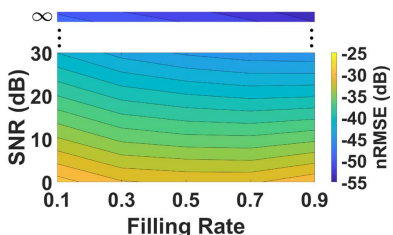

(a)

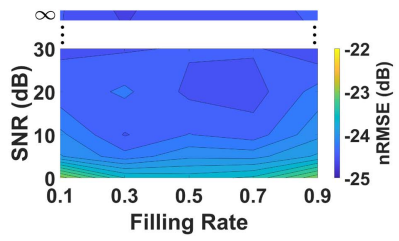

(c)

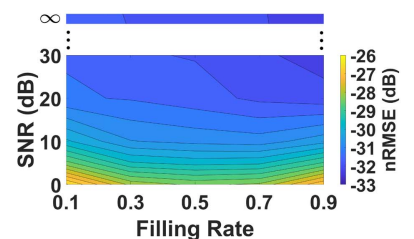

(b)

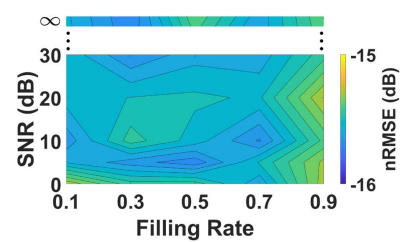

(d)
Fig. 6. Contour plots of the nRMSE of the estimated system matrices for different filling rates $(\nu)$ and $\delta$ values for R-CCS. For $\nu$, we used the following values: $0.1,0.3,0.5,0.7$, and 0.9 . a) $\delta=0.45$. b) $\delta=0.18$. c) $\delta=0.1$. d) $\delta=0.05$.

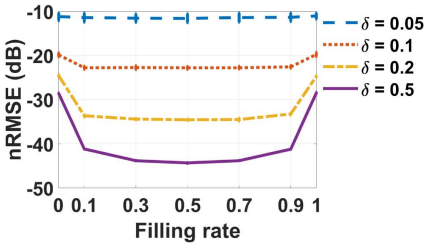

(a)

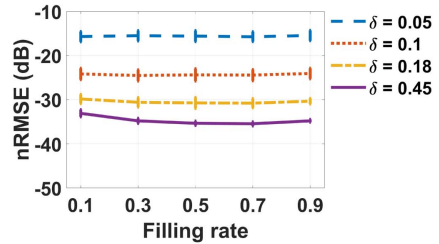

(b)
Fig. 7. The $n R M S E(d B)$ as a function of filling rate $(\nu)$ for $10 \mathrm{~dB}$ SNR for a) M-CCS, and b) R-CCS. The mean values and standard deviations across repeated Monte Carlo simulations are plotted.

values given in Fig. 5 and Fig. 6, respectively. In addition, we present cross sections of these plots for $10 \mathrm{~dB}$ SNR in Fig. 7. For M-CCS, the results show that for $\delta=0.5$ and $\delta=0.2$ cases, the best $v$ is 0.5 , independent of the SNR level. At lower measurement rates of $\delta=0.1$ and $\delta=0.05$, the effect of $v$ is much less prominent, as clearly shown in Fig. 7a. At $\delta=0.1$, while the best $v$ is 0.3 , $v$ values between $0.1-0.9$ also have comparable performances. At $\delta=0.05$, the contour plot in Fig. $5 \mathrm{~d}$ is not monotonous along the vertical direction due to both the reduced effect of $v$ and the high standard deviation of the MC simulations (see the error bars in Fig. 7a). For the optimal $v$ values at $10 \mathrm{~dB}$ SNR, nRMSE is improved by $16 \mathrm{~dB}, 10 \mathrm{~dB}, 3 \mathrm{~dB}$, and $0.5 \mathrm{~dB}$ compared to standard CS (i.e., $v=1 / 800$ ) for $\delta=0.5$, $0.2,0.1$, and 0.05, respectively (Fig. 7a). As seen in Fig. 5, for all four measurement rates, choosing the correct $v$ causes even more significant improvements in nRMSE at SNRs lower than $10 \mathrm{~dB}$.

For R-CCS robustness analysis, the results are given in Fig. 6 and Fig. 7b. Note that a single-voxel MNP version and its dual case (i.e., $v=1 / 800$ and $v=799 / 800$ ) were not included, as they are not feasible with the R-CCS implementation. These results indicate that for $\delta=0.45$, the best $v$ is 0.7 , whereas for $\delta=0.18$, it is 0.5 . Similar to M-CCS, the effect of $v$ is less significant at lower measurement rates (see Fig. 7b). At $\delta=0.1, v$ values between $0.3-0.7$ yield similar performances. At $\delta=0.05$, all $v$ values between

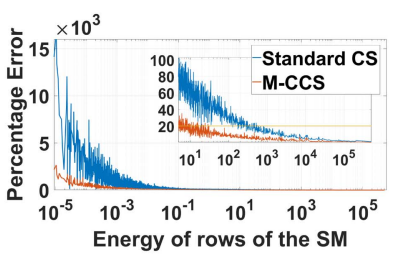

(a)

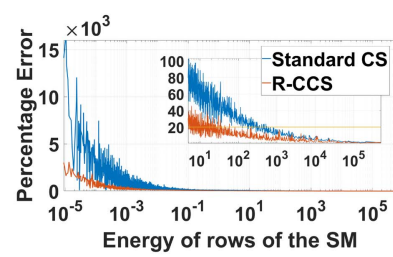

(b)
Fig. 8. The percentage error as a function of energy of rows of the SM for a) M-CCS, and b) R-CCS with parameters: $\nu=0.1, \delta=0.2$, and $10 \mathrm{~dB}$ SNR.
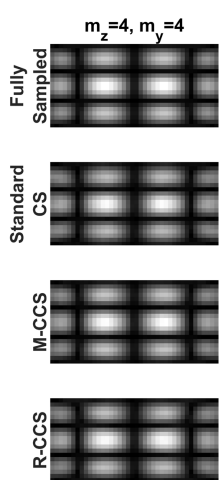
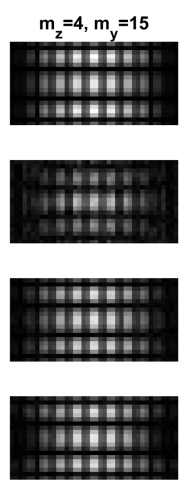
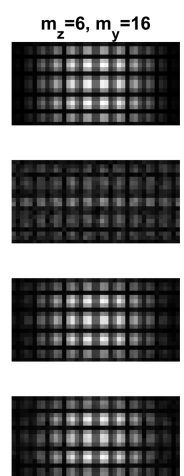
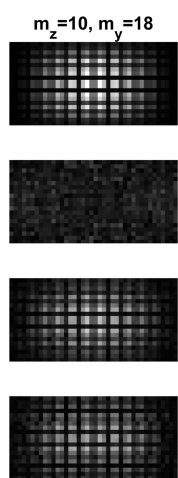

Fig. 9. Selected rows of the reconstructed SMs. The top row shows the fully sampled ideal case as reference without the additive noise. The rows estimated with M-CCS (third row) and R-CCS (bottom row) have finer structures than the ones estimated with the standard CS case (second row) for parameters: $\nu=0.1, \delta=0.2$, and $10 \mathrm{~dB}$ SNR.

0.1-0.9 yield similar SM reconstruction performances, causing the contour plot to be dominated by the standard deviation of the MC simulations.

These SM reconstruction analyses show that a significant improvement over the standard CS method is obtained for $v$ ranging between $0.1-0.9$. Note that a $v$ of 0.1 has the added practical advantage of requiring a smaller volume of MNPs. For both M-CCS and R-CCS, $v$ has a smaller effect at lower $\delta$ values, i.e., for highly accelerated calibrations.

\section{B. Row-wise Analysis of SM Reconstruction Performance}

Figure 8 shows the percentage error as a function of energy of rows of the SM for M-CCS and R-CCS (with a comparison to the standard CS method) with parameters $v=0.1$, $\delta=0.2$, and $10 \mathrm{~dB}$ SNR. When the standard CS method was used for SM reconstruction, 5.3\% of the SM rows were reconstructed with a relative error less than $20 \%$. This rate is increased to $17.2 \%$ for the M-CCS, and $14.6 \%$ for the R-CCS methods.

In Fig. 9, some selected rows of the reconstructed SMs are presented for standard CS method, M-CCS, and R-CCS. The fully sampled ideal rows with infinite SNR are also shown for comparison. As clearly seen, the quality of the rows reconstructed with the standard CS method reduces rapidly for high mixing orders, whereas the rows reconstructed with M-CCS and R-CCS preserve their structures. 


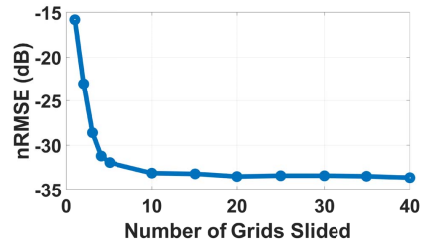

(a)

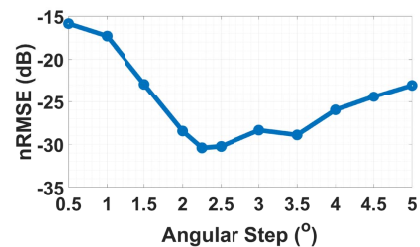

(b)
Fig. 10. The nRMSE (dB) as a function of a) the number of slided grids $\left(\Delta_{y}\right)$ for S-CCS, and b) the angular step size $\left(\Delta_{\theta}\right)$ for R-CCS before each measurement with parameters: $\nu=0.1, \delta=0.2$, and $10 \mathrm{~dB}$ SNR.

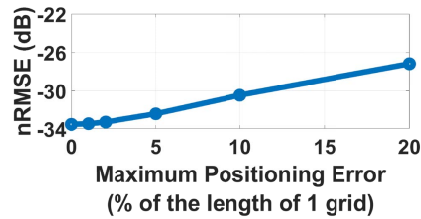

(a)

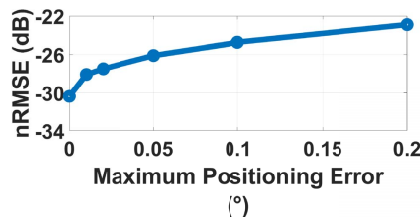

(b)
Fig. 11. The effect of random positioning error on the nRMSE for a) S-CCS and b) R-CCS with parameters: $\nu=0.1, \delta=0.2$, and $10 \mathrm{~dB}$ SNR.

\section{Effects of Sliding Step Size $\left(\Delta_{\mathrm{y}}\right)$ and Angular Step Size $\left(\Delta_{\theta}\right)$}

For S-CCS, we show the effect of $\Delta_{y}$ on SM reconstruction in Fig. 10a. In this analysis, the FOV size was $40 \times 20$ pixels with $\delta=0.2$, corresponding to a total of 160 calibration measurements. Therefore, sliding along the y-direction with $\Delta_{y}=1$ grid before each measurement required an S-CCS with a total length of 199 grids $\left(N_{y}+\Delta_{y}(\delta N-1)\right)$, whereas using a $\Delta_{y}=40$ grids corresponded to fully replacing the effective CCS before each measurement (i.e., equivalent to M-CCS), and required an S-CCS with a length of 6400 grids. The results in Fig. 10a show that nRMSE for SM reconstruction decays sharply from one to four grid steps, emphasizing the need for a sufficient level of variation among the effective regions of S-CCS that fall within the FOV. On the other hand, performance remains almost unaffected when we increase $\Delta_{y}$ from 10 to 40 grids.

For R-CCS, we show the effect of $\Delta_{\theta}$ in Fig. 10b. In this analysis, with the total number of calibration measurements fixed to $160, \Delta_{\theta}$ ranged from $0.5^{\circ}$ to $5^{\circ}$. The results show that increasing $\Delta_{\theta}$ up to $2.25^{\circ}$ improves the performance significantly, once again emphasizing the need for variation among effective portions of the R-CCS. However, for $\Delta_{\theta}$ values larger than $2.25^{\circ}$, R-CCS rotates more than $360^{\circ}$ in total. Without incorporating a sliding movement, the measurements exceeding one full turn result in redundant and/or similar measurements increasing the reconstruction error as given in Fig. 10b.

\section{Effects of Positioning Error}

In Fig. 11a, we plotted nRMSE of SM reconstruction for S-CCS with $\Delta_{y}=40$ grids as a function of positioning error for $\delta=0.2, v=0.1$, and $10 \mathrm{~dB}$ SNR. For this scenario, to limit the increase in the reconstruction error to less than $1 \mathrm{~dB}$, the random positioning error should be smaller than $\sim 5 \%$ of

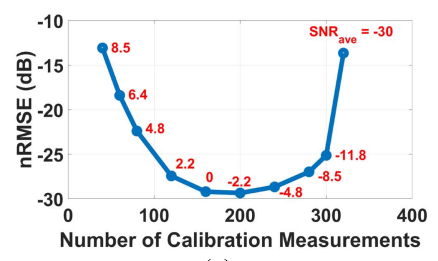

(a)

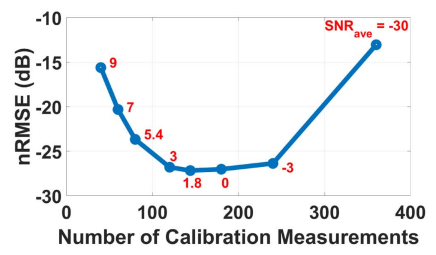

(b)
Fig. 12. Performance under limited calibration time. The nRMSE and SNR after averaging (SNR ave, given in $\mathrm{dB}$ and shown in red) as a function of the total number of measurements for a) S-CCS with 40-grid step size, and b) R-CCS, both with $\nu=0.1$.

the length of 1 grid, such that for a uniform distribution the positioning error stays within $\pm 5 \%$ of the length of 1 grid from the true position. Even though the nRMSE increases by $6.33 \mathrm{~dB}$ at $20 \%$ positioning error, it still remains $2.54 \mathrm{~dB}$ better than nRMSE for the standard CS method at the same measurement rate and SNR.

In Fig. 11b, we plotted nRMSE of SM reconstruction for $\mathrm{R}-\mathrm{CCS}$ as a function of angular positioning error for $\delta=0.2$, $v=0.1$, and $10 \mathrm{~dB}$ SNR. For this scenario, up to a maximum angular positioning error of $0.01^{\circ}$, the reconstruction error is still better than that of the standard CS method.

\section{E. Performance Under a Limited Calibration Time Constraint}

Figure 12 shows the results of the limited calibration time analysis, clearly demonstrating the trade-off between using the time budget to increase the SNR via averaging vs. to vary the effective CCS. For the results we show in Fig. 12a, we used an S-CCS with $\Delta_{y}=40$ grids with the total calibration time fixed to $\sim 320$ seconds. Hence, when we reduced the number of calibration measurements, we increased the time allocated for each measurement. For instance, when 160 calibration measurements were used instead of 320 , the allocated scan time for each measurement was increased to 2 seconds. With the assumption that 1 second is required for the mechanical movement, the remaining 1 second was used to acquire 1000 Lissajous scans from the same scene. Averaging those acquisitions increased the SNR by $30 \mathrm{~dB}$, so that the SNR after averaging $\left(\mathrm{SNR}_{\mathrm{ave}}\right)$ became $0 \mathrm{~dB}$ (see red labels in Fig. 12a). We give the nRMSE and the corresponding $S_{N R}$ ave levels as a function of the number of calibration measurements in Fig. 12a. These results show that the optimal strategy is to set the number of calibration measurements as high as possible, while simultaneously ensuring sufficient $\mathrm{SNR}_{\text {ave }}$ for each measurement. For the analyzed scenario, we reached the optimal trade-off for 200 measurements $(\delta=0.25)$, where $\mathrm{SNR}_{\mathrm{ave}}$ is $-2.2 \mathrm{~dB}$.

As we show in Fig. 12b, we obtained similar results for R-CCS, where we fixed the total calibration time to $\sim 360$ seconds. The optimal number of measurements is 144 for this case (i.e., $\delta=0.18$ ), where $\mathrm{SNR}_{\text {ave }}$ is $1.8 \mathrm{~dB}$.

\section{F. Image Reconstruction Results}

We present the images reconstructed using the resultant SMs of standard CS, M-CCS, S-CCS, R-CCS, RS-CCS, and 
Standard CS Method

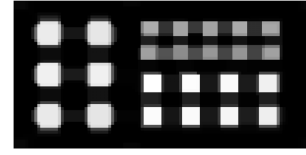

\section{$\mathrm{M-CCS}$}

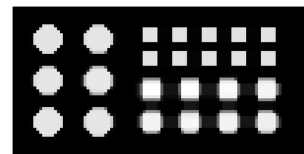

S-CCS

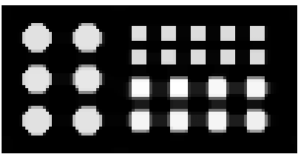

R-CCS

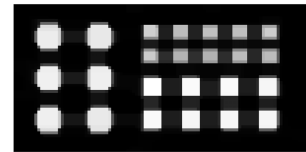

RS-CCS

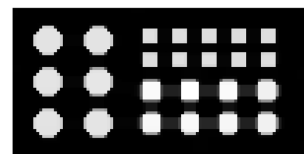

RSC-CCS

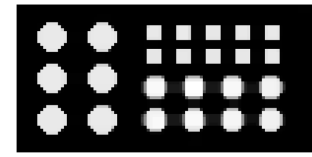

(a)

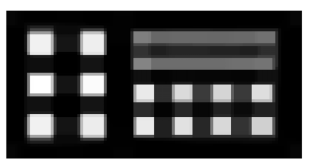

Proposed Methods
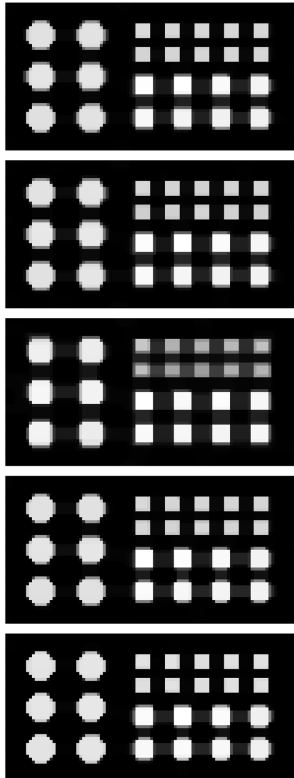

(b)
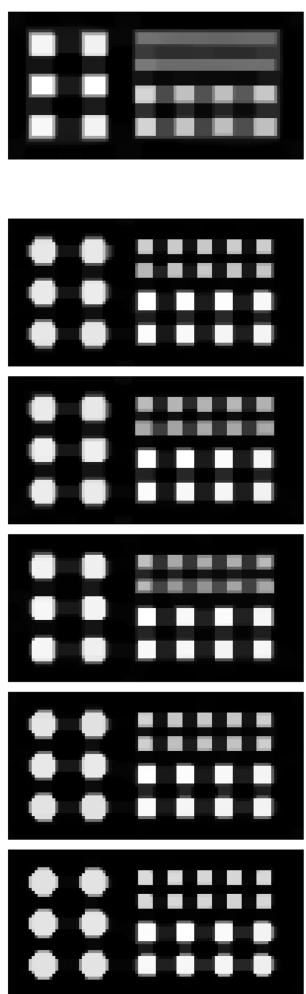

(c)

Fig. 13. Reconstructed images with system matrices reconstructed via the standard CS method, M-CCS, S-CCS (with $\Delta_{y}=5$ grids), R-CCS, RS-CCS, and RSC-CCS, for a) $\delta=0.2$ and $10 \mathrm{~dB}$ SNR, b) $\delta=0.2$ and $0 \mathrm{~dB}$ SNR, and c) $\delta=0.1$ and $0 \mathrm{~dB}$ SNR scenarios. Images are shown with identical windowing. $\nu$ of the CCSs were 0.1 .

TABLE |

SSIMS OF RECONSTRUCTED IMAGES $(\nu=0.1)$

\begin{tabular}{|c|c|c|c|}
\hline Methods & $\begin{array}{c}\delta=\mathbf{0 . 2} \\
\mathbf{1 0} \mathbf{~ d B ~ S N R}\end{array}$ & $\begin{array}{c}\delta=\mathbf{0 . 2} \\
\mathbf{0} \text { dB SNR }\end{array}$ & $\begin{array}{c}\delta=\mathbf{0 . 1} \\
\mathbf{0} \text { dB SNR }\end{array}$ \\
\hline Standard CS & 0.71 & 0.55 & 0.49 \\
\hline M-CCS & 0.94 & 0.88 & 0.81 \\
\hline S-CCS & 0.91 & 0.86 & 0.74 \\
\hline R-CCS & 0.78 & 0.73 & 0.70 \\
\hline RS-CCS & 0.93 & 0.89 & 0.83 \\
\hline RSC-CCS & 0.97 & 0.94 & 0.90 \\
\hline
\end{tabular}

RSC-CCS methods in Fig. 13 for the phantom in Fig. 4 with a FOV size of $100 \times 50$ voxels.

For all scenarios in Fig. 13, all CCS outperforms the standard CS method, as evident by both visual inspection and the quantitative results in Tables I and II. For instance, for $\delta=0.1$ and $0 \mathrm{~dB}$ SNR, the SSIM and PSNR values increase by 0.41 and $5.7 \mathrm{~dB}$, respectively, for RSC-CCS implementation. All of the CCS-based methods, except R-CCS, successfully resolved the smaller square objects at the top right part of the numerical phantom, whereas the standard CS method fell short. R-CCS did not resolve the smaller objects as successfully as other CCS methods, as a result of high mutual coherence among measurements. Although the randomness of the CCS is partially violated for the RSC-CCS, the corresponding reconstructions shown in Fig. 13 have the highest quality.
TABLE ॥

PSNRS OF RECONSTRUCTED IMAGES $(\nu=0.1)$

\begin{tabular}{|c|c|c|c|}
\hline Methods & $\begin{array}{c}\delta=\mathbf{0 . 2} \\
\mathbf{1 0} \mathbf{~ d B ~ S N R}\end{array}$ & $\begin{array}{c}\delta=\mathbf{0 . 2} \\
\mathbf{0} \mathbf{~ d B} \text { SNR }\end{array}$ & $\begin{array}{c}\delta=\mathbf{0 . 1} \\
\mathbf{0} \text { dB SNR }\end{array}$ \\
\hline Standard CS & 13.64 & 12.04 & 11.63 \\
\hline M-CCS & 19.72 & 16.94 & 15.26 \\
\hline S-CCS & 18.00 & 16.28 & 14.16 \\
\hline R-CCS & 14.55 & 13.90 & 13.51 \\
\hline RS-CCS & 18.84 & 17.16 & 15.42 \\
\hline RSC-CCS & 22.12 & 19.14 & 17.32 \\
\hline
\end{tabular}

For S-CCS, we show the effects of $\Delta_{y}$ on the reconstructed images in Fig. 14. While sliding only a single grid after each measurement yielded a blurred image, sliding 5 grids per step was sufficient to reach a high-quality reconstruction. In addition, sliding 10 grids per step resulted in nearly identical image quality as sliding 5 grids per step. This result is consistent with the SM reconstruction analysis in Fig. 10a, and highlights the fact that the effective CCS needs to vary among measurements to ensure that each measurement provides maximal amount of new additional information.

\section{DISCUSSION}

The proposed CCS framework results in significantly improved image quality compared to the standard CS method for the same SNR and measurement rate. This is a consequence of (i) the higher effective SNR of each CCS 


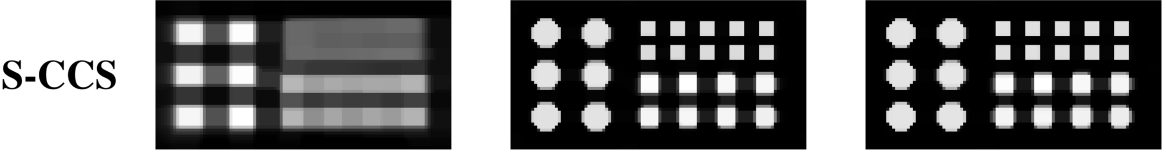 \\ (a) \\ (b) \\ (c)}

Fig. 14. Image reconstruction results for S-CCS with $\Delta_{y}$ values a) 1 grid, b) 5 grids, and c) 10 grids. $\delta=0.2,0 \mathrm{~dB}$ SNR, and $\nu=0.1$. Images are shown with identical windowing.

measurement compared to single-voxel MNP measurements, and (ii) the higher amount of information gathered from a single measurement. In the literature, several other techniques were presented to improve the SNR and measurement time of SM measurements. One such technique proposed a hybrid approach for performing calibrations in a separate device emulating the MPI system [14]. Although this method decreases calibration time with high SNR measurements, system imperfections such as the inhomogeneity of the magnetic fields, analog and digital perturbations of the MPI system are not present in the measurements. Another method decreases the SF gradients to enhance the SNR by increasing the amount of responsive MNPs [19]. The increase in the SNR is limited by the maximum number of MNPs that can be placed inside one voxel in this case. The proposed CCS approach directly measures the SM under the effects of the aforementioned nonidealities, with a relatively high SNR and in a convenient measurement duration.

The filling rate analyses show that the optimal $v$ is between 0.1 and 0.9. While SNR increases with increasing $v$ above 0.5 , the coherency of the measurements also increases, limiting the SM reconstruction performance. The symmetrical behaviour with respect to $v$ shows that the reconstruction performance is limited by the ill-posedness of the problem, rather than the measurement noise. Furthermore, the effect of measurement noise on the SM reconstruction is reduced at lower $\delta$ values. In this work, we tested the image reconstruction performances for $v=0.1$. We chose this $v$ as it provides a significant improvement over the standard CS method, while requiring a relatively small volume of MNPs. Higher $v$ values can provide further but modest improvements over $v=0.1$. For example, with $v=0.3$ at $\delta=0.2$ and $10 \mathrm{~dB}$ SNR, the SSIM and PSNR values for the images reconstructed for M-CCS are improved by 0.02 and $1.3 \mathrm{~dB}$, respectively (results not shown). $v$ values of 0.3 and 0.5 , on the other hand, result in nearly identical image quality metrics. These improvements over $v=0.1$ are gradually reduced for more difficult cases with reduced SNR and smaller $\delta$.

The CCS framework is based on measuring multiple "scenes" with randomly positioned MNPs, i.e., M-CCS. We proposed other CCS variations from a practical implementation point of view. While we place the MNP samples at random positions in S-CCS and R-CCS, the successive measurements become correlated during sliding and rotating movements. Although the CS conditions to collect random and incoherent measurements are partly violated, our analyses on slided grid and angular step sizes suggest that the SM is sufficiently sparse [17] to enable successful SM reconstructions even in these implementations. For example, we can slide a long CCS with 4-5 grid step size between measurements to enable successful SM reconstructions for a 40-grid FOV and a measurement rate of 0.2 . Note, however, the practicality of the particular CCS implementation is a separate issue. In this case, a large CCS that is at least 20 times the size of the FOV is required.

We proposed the R-CCS implementation to mitigate the large size requirement of S-CCS. However, using only rotations resulted in reduced $\mathrm{SM}$ reconstruction performance compared to M-CCS, despite using an R-CCS with a size 12.5 times the FOV. As a solution, we proposed the RS-CCS implementation to combine rotations and translations, so that the measurements could be diversified. For example, we showed RS-CCS with only 2-3 rotation centers result in a similar imaging performance to M-CCS and S-CCS. For closed-bore MPI scanners, the diameter of R-CCS and its variations cannot be larger than that of the bore. The range of the sliding movement is also limited in that case. Therefore, for the scanners that have a small bore, S-CCS option may be preferred.

In MPI, MNPs outside the DF-FOV can induce signal in the receiver coils, causing artefacts along the edges of the image [34]. For calibration using a single MNP sample, these artefacts can be mitigated by calibrating the SM for a larger FOV than the DF-FOV [35]. However, the calibration time would considerably increase in that case. In contrast, S-CCS and R-CCS implementations are already larger than the DF-FOV. Therefore, one can easily reconstruct a larger SM to take into account the response of the MNPs that are outside the DF-FOV. Importantly, this procedure would not cause any increase in the calibration duration, as these CCSs already contain MNP samples outside the DF-FOV.

In CS methods, the number of measurements are fewer than the total number of grids. Therefore, the $C$ matrix in Eqn. (2), which represents the CCSs, is not invertible. Here, we designed full column rank $C$ matrices. This design ensures having linearly independent measurements that maximize information content.

In this work, we used the DCT domain for a sparse reconstruction of the SM. The proposed method can also be adapted to other sparse transformation domains by simply replacing the matrix $D$ in Eqn. (2) with the corresponding transformation matrices. For example, when Chebyshev transformation is used for sparsity representation [36], the amount of MNPs positioned in non-equidistant and non-equally sized grids required for Chebyshev transformation can be measured and calculated. SM and image reconstruction can be performed based on that new gridding.

We used the ADMM algorithm to solve the optimization problems of SM and image reconstructions. The time required for SM reconstruction was 933 seconds 
(300 ADMM iterations), whereas it was 96 seconds for image reconstruction (100 ADMM iterations) for $100 \times$ 50 grids using a standard workstation having 2 Intel ${ }^{\circledR}$ Xeon ${ }^{\circledR}$ E5-2620 v3 CPUs and 64 GB RAM. SM reconstruction time for the standard CS method is also similar, since the problem definition in Eqn. (2) applies for both cases. Accordingly, SM reconstruction time depends on the number of frequency components and the $\delta$. With that said, CCS approach may yield faster convergence compared to the standard CS due to its improved SNR. While the computational load of ADMM is relatively high, we previously showed that the speed of the ADMM algorithm for MPI image reconstruction can be accelerated using GPUs [37]. In that study, a 40-fold acceleration was achieved with a single NVIDIA® GeForce GTX 1080. As ADMM is a highly parallelizable algorithm, even higher accelerations are expected using multiple GPUs.

One of the main advantages of the CCS framework is the increased SNR of the calibration measurements, which reduces the need for signal averaging. Accordingly, calibration data can be acquired during a continuous movement of the CCS, which would significantly reduce the calibration duration at the risk of introducing movement artefacts. The speed of the movement should be adjusted and the calibration data should be acquired in a relatively short time interval, such that the position change of the MNP samples do not degrade the SM reconstruction. One can optimize the level of signal averaging, the measurement rate, and the speed of the continuous movement according to the measurement SNR.

For continuous motion, we would need to know the position of the CCS precisely to accurately generate the calibration masking matrix, $C$. Our analysis on positioning errors shows that for $5 \%$ positioning error, nRMSE of SM reconstruction increases only marginally by $1 \mathrm{~dB}$ at $\delta=0.2, v=0.1$, and $10 \mathrm{~dB}$ SNR. To fully compensate for positioning errors, we can track the position of the CCS in real time using precision position measurement devices such as laser trackers.

Implementing the CCS as a mold that can be filled with MNP samples may prove challenging to fill and empty the random CCS pattern with MNP samples. Here, we proposed the RSC-CCS implementation featuring a contiguous CCS pattern as a solution. To minimize the size, it incorporates a combination of rotations and translations. The imaging results show that this implementation has the highest image quality among all proposed implementations. The voxels with MNPs are contiguous in this case rather than being randomly distributed, which improved the signal power during the scan. Experimental demonstration of this practical approach remains a future work.

Practically, fabrication errors can affect the success of the calibrations using CCS. Errors from imprecise CCS fabrication can be mitigated by imaging the CCS using a CT/micro-CT scanner to determine the exact positions of MNPs. For contiguous CCSs, additional precautions must be taken in order not to introduce air bubbles in the nanoparticle channels, as bubble movement might cause variations in MNP distribution over time. In this work, nanoparticle concentration was assumed to be constant at all MNP positions. The reconstruction performance can deteriorate if nanoparticle density fluctuates throughout the CCS.

Another practical consideration may be the amount of tracer material used in CCSs. For the CCS parameters used in Fig. $13 \mathrm{~b}\left(100 \times 50\right.$ pixels, $\delta=0.2, v=0.1$, and $0.25 \mathrm{~mm}^{3}$ grid size), the required tracer volumes can be calculated as $125 \mathrm{ml}$ for M-CCS, $6.37 \mathrm{ml}$ for S-CCS, and $1.56 \mathrm{ml}$ for R-CCS and its variations. Hence, R-CCS and its variations are also advantageous in this sense.

In this work, the CCS method was analyzed for the 2D case, however, the same principles can easily be extended to the 3D case. For M-CCS and S-CCS, the CCS can be implemented as a rectangular prism, and S-CCS can slide in any direction in space. For R-CCS and its variations, the CCS can be implemented as a sphere that can slide in any direction and rotate around any axis.

\section{CONCLUSION}

We proposed a fast calibration method for the system matrix in MPI by combining CCS-based measurements with CS recovery. We demonstrated five different practical implementations of CCS for realistic imaging scenarios. The results show that MPI system calibration using CCS outperforms the standard CS method, which uses a single-voxel MNP sample. CCS enhances the quality of reconstructed SM and MPI images for the same measurement rate and SNR. Therefore, CCS can substantially reduce calibration time, and further improvements may be viable by considering on-the-move data acquisitions.

\section{ACKNOWLEDGMENT}

E. U. Saritas and H. E. Güven share senior authorship. The authors would like to thank Mert Kalfa for his valuable contributions to the discussions of the intermediate results.

\section{ReFERENCES}

[1] B. Gleich and J. Weizenecker, "Tomographic imaging using the nonlinear response of magnetic particles," Nature, vol. 435, no. 7046, pp. 1214-1217, Jun. 2005.

[2] J. Haegele et al., "Magnetic particle imaging: Visualization of instruments for cardiovascular intervention," Radiology, vol. 265, no. 3, pp. 933-938, Dec. 2012.

[3] B. Zheng et al., "Quantitative magnetic particle imaging monitors the transplantation, biodistribution, and clearance of stem cells in vivo," Theranostics, vol. 6, no. 3, pp. 291-301, 2016.

[4] J. Yang, J. Gunn, S. R. Dave, M. Zhang, Y. Wang, and X. Gao, "Ultrasensitive detection and molecular imaging with magnetic nanoparticles," Analyst, vol. 133, no. 2, pp. 154-160, 2008.

[5] J. R. McCarthy and R. Weissleder, "Multifunctional magnetic nanoparticles for targeted imaging and therapy," Adv. Drug Del. Rev., vol. 60, no. 11, pp. 1241-1251, Aug. 2008.

[6] M. Grüttner et al., "On the formulation of the image reconstruction problem in magnetic particle imaging," Biomedizinische Technik/Biomed. Eng., vol. 58, no. 6, pp. 583-591, 2013.

[7] T. Knopp et al., "Model-based reconstruction for magnetic particle imaging," IEEE Trans. Med. Imag., vol. 29, no. 1, pp. 12-18, Jan. 2010.

[8] P. W. Goodwill and S. M. Conolly, "Multidimensional x-space magnetic particle imaging," IEEE Trans. Med. Imag., vol. 30, no. 9, pp. 1581-1590, Sep. 2011.

[9] P. W. Goodwill and S. M. Conolly, "The X-space formulation of the magnetic particle imaging process: 1-D signal, resolution, bandwidth, SNR, SAR, and magnetostimulation," IEEE Trans. Med. Imag., vol. 29, no. 11, pp. 1851-1859, Nov. 2010. 
[10] T. Knopp et al., "Weighted iterative reconstruction for magnetic particle imaging," Phys. Med. Biol., vol. 55, no. 6, pp. 1577-1589, Feb. 2010.

[11] M. Storath et al., "Edge preserving and noise reducing reconstruction for magnetic particle imaging," IEEE Trans. Med. Imag., vol. 36, no. 1, pp. 74-85, Jan. 2017.

[12] S. Ilbey, C. B. Top, A. Güngör, T. Cukur, E. U. Saritas, and H. E. Güven, "Comparison of system-matrix-based and projection-based reconstructions for field free line magnetic particle imaging," Int. J. Magn. Particle Imag., vol. 3, no. 1, 2017, Art. no. 1703022.

[13] M. Graeser, K. Bente, A. Neumann, and T. M. Buzug, "Trajectory dependent particle response for anisotropic mono domain particles in magnetic particle imaging," J. Phys. D, Appl. Phys., vol. 49, no. 4, Feb. 2016, Art. no. 045007

[14] A. von Gladiss, M. Graeser, P. Szwargulski, T. Knopp, and T. M. Buzug, "Hybrid system calibration for multidimensional magnetic particle imaging," Phys. Med. Biol., vol. 62, no. 9, p. 3392, May 2017.

[15] J. Rahmer, J. Weizenecker, B. Gleich, and J. Borgert, "Signal encoding in magnetic particle imaging: Properties of the system function," $B M C$ Med. Imag., vol. 9, no. 1, p. 4, Apr. 2009.

[16] J. Lampe et al., "Fast reconstruction in magnetic particle imaging," Phys. Med. Biol., vol. 57, no. 4, pp. 1113-1134, Feb. 2012.

[17] T. Knopp and A. Weber, "Sparse reconstruction of the magnetic particle imaging system matrix," IEEE Trans. Med. Imag., vol. 32, no. 8, pp. 1473-1480, Aug. 2013

[18] A. Weber and T. Knopp, "Reconstruction of the magnetic particle imaging system matrix using symmetries and compressed sensing," Adv. Math. Phys., vol. 2015, Sep. 2015, Art. no. 460496.

[19] M. Graeser, A. von Gladiss, T. Friedrich, and T. M. Buzug, "SNR and discretization enhancement for system matrix determination by decreasing the gradient in magnetic particle imaging," Int. J. Magn. Part. Imag., vol. 3, no. 1, 2017, Art. no. 1703019.

[20] G. R. Arce, D. J. Brady, L. Carin, H. Arguello, and D. S. Kittle, "Compressive coded aperture spectral imaging: An introduction," IEEE Signal Process. Mag., vol. 31, no. 1, pp. 105-115, Jan. 2014.

[21] S. Ilbey, C. B. Top, E. U. Saritas, and H. E. Güven, "Fast system calibration for MPI using a rotating coded calibration scene," in Proc. 8th Int. Workshop Magn. Part. Imag. (IWMPI), Hamburg, Germany, Mar. 2018, pp. 63-64.

[22] E. J. Candès, Y. C. Eldar, D. Needell, and P. Randall, "Compressed sensing with coherent and redundant dictionaries," Appl. Comput. Harmon. Anal., vol. 31, no. 1, pp. 59-73, Jul. 2011.

[23] T. Knopp et al., "Trajectory analysis for magnetic particle imaging," Phys. Med. Biol., vol. 54, no. 2, pp. 385-397, Jan. 2009.

[24] R. M. Ferguson et al., "Magnetic particle imaging with tailored iron oxide nanoparticle tracers," IEEE Trans. Med. Imag., vol. 34, no. 5, pp. 1077-1084, May 2015.
[25] A. P. Khandhar et al., "Evaluation of PEG-coated iron oxide nanoparticles as blood pool tracers for preclinical magnetic particle imaging," Nanoscale, vol. 9, no. 3, pp. 1299-1306, 2017.

[26] T. Knopp and T. M. Buzug, Magnetic Particle Imaging: An Introduction to Imaging Principles and Scanner Instrumentation. Berlin, Germany: Springer, 2012

[27] M. V. Afonso, J. M. Bioucas-Dias, and M. A. T. Figueiredo, "An augmented Lagrangian approach to the constrained optimization formulation of imaging inverse problems," IEEE Trans. Image Process., vol. 20, no. 3, pp. 681-695, Mar. 2011

[28] S. J. Wright, R. D. Nowak, and M. A. T. Figueiredo, "Sparse reconstruction by separable approximation," IEEE Trans. Signal Process., vol. 57, no. 7, pp. 2479-2493, Jul. 2009.

[29] J. A. Tropp and A. C. Gilbert, "Signal recovery from random measurements via orthogonal matching pursuit," IEEE Trans. Inf. Theory, vol. 53, no. 12, pp. 4655-4666, Dec. 2007.

[30] S. Boyd, N. Parikh, E. Chu, B. Peleato, and J. Eckstein, "Distributed optimization and statistical learning via the alternating direction method of multipliers," Found. Trends Mach. Learn., vol. 3, no. 1, pp. 1-122, Jan. 2011.

[31] H. Güven, A. Güngör, and M. Çetin, "An augmented Lagrangian method for complex-valued compressed sar imaging," IEEE Trans. Comput. Imag., vol. 2, no. 3, pp. 235-250, Sep. 2016.

[32] S. Ilbey, C. B. Top, T. Çukur, E. U. Saritas, and H. E. Güven, "Image reconstruction for magnetic particle imaging using an augmented Lagrangian method," in Proc. IEEE 14th Int. Symp. Biomed. Imag., Melbourne, VIC, Australia, Apr. 2017, pp. 64-67.

[33] Z. Wang, A. C. Bovik, H. R. Sheikh, and E. P. Simoncelli, "Image quality assessment: From error visibility to structural similarity," IEEE Trans. Image Process., vol. 13, no. 4, pp. 600-612, Apr. 2004.

[34] J. Rahmer et al., "Results on rapid 3D magnetic particle imaging with a large field of view," in Proc. Intl. Soc. Mag. Reson. Med., Montreal, Canada, vol. 19, May 2011, p. 629.

[35] A. Weber, F. Werner, J. Weizenecker, T. M. Buzug, and T. Knopp, "Artifact free reconstruction with the system matrix approach by overscanning the field-free-point trajectory in magnetic particle imaging," Phys. Med. Biol., vol. 61, no. 2, pp. 475-487, Jan. 2016.

[36] L. Schmiester and M. Möddel, W. Erb, and T. Knopp, "Direct image reconstruction of lissajous-type magnetic particle imaging data using chebyshev-based matrix compression," IEEE Trans. Comput. Imag., vol. 3 , no. 4, pp. 671-681, Dec. 2017.

[37] S. Ilbey, A. GüngÃ̃f[ör, C. B. Top, E. Ü. Saritaş, and H. E. Güven, "Real-time three-dimensional image reconstruction using alternating direction method of multipliers for magnetic particle imaging," in Proc. 26th Signal Process. Commun. Appl. Conf. (SIU), Izmir, Turkey, May 2018, pp. 1-4. 